\title{
Related research and language study
}

that school policies must balance the rights of individuals, especially rights to education in one's own language, with practicalities and future needs. This can lead to various choices, ranging from a single language of schooling for an entire country to separate schools for each language community. To be used as a medium in schools, a language has to fulfil certain criteria: wide and up-to-date vocabulary, adequate phonological and grammatical description, good writing system, and extensive and varied literature; languages can be helped towards this status, a process Calvet calls équipement de la langue. A practical programme is outlined for implementing new language policies in schools, especially in developing countries, with a checklist of procedures and a flowchart to allow for different conditions. Different kinds of bilingualism and their educational effects are also discussed.

\section{7-267 Wiley, Terrence G. and Lukes, Marguerite (California State U.). English-only}

and standard English ideologies in the U.S. TESOL Quarterly (Alexandria, VA), 30, 3 (1996), 511-35.

This article probes assumptions underlying dominant U.S. ideologies regarding language diversity (both between English and other languages and among varieties of English) and their impact on language planning and policy from a historical-structural perspective by analysing and synthesising a broad base of literature. It compares and contrasts two popularly accepted ideologies. The first is the ideology of English monolingualism, which the authors see as framing policy issues in an immigrant paradigm in order to portray language diversity as an alien and divisive force; the second involves a standard language ideology that is used to position speakers of different varieties of the same language within a social hierarchy. The article discusses the connection between assumptions underlying linguistic ideologies and other social ideologies related to individualism and social mobility through education. It discusses limitations in the immigrant paradigm and considers the instrumental role that schools play in positioning students by using language assessment and classification schemes. Dilemmas and opportunities for contesting these ideologies are addressed.

\section{7-268 Zimmer, Armand (Cross-border Documentation and Training Centre for Learning Neighbouring Languages). Les langues aux frontières: l'exemple de la coopération transfrontalière de proximité Moselle-Sarre. [Language across frontiers: Moselle-Sarre crossborder cooperation.] Etudes de Linguistique Appliquée (Paris), 103 (1996), 267-84.}

The Department of Moselle in France and the Land of Sarre in Germany are cooperating to develop programmes specially designed to establish FrancoGerman bilingualism in the Moselle-Sarre border region. The programmes are based on the recognition and fostering of a specific regional identity, taking into account the characteristics peculiar to each of the partners and the constraints to which they are subject, and maximising the advantages afforded by their close proximity. The education authorities of both regions have equipped themselves to achieve the common goal by setting up the appropriate structures. Cross-border cooperation in the field of education strengthens action already taken. Local communities in particular are actively supporting the project.

\section{Pragmatics}

97-269 Davidson, Brad (Stanford U., CA). 'Pragmatic weight' and Spanish subject pronouns: The pragmatic and discourse uses of 'tú' and 'yo' in spoken Madrid Spanish. Journal of Pragmatics (Amsterdam), 26, 4 (1996), 543-65.

Spanish is a pro-drop language in which first and second person subject pronouns are frequently omitted (80\%); traditionally the function of overt subject pronouns has been regarded as adding emphasis or to contrast explicit statements. This paper argues that their function is more similar to that of topicalised noun phrases, referred to as XForms by Klein-Andreu, and that their appearance is governed by their conversational function as discourse topics; they are used pragmatically and meta-linguistically to switch reference, for purposes of emphasis and negotiating conversational turns, and to add 'pragmatic weight' to frames of reference, epistemic parentheticals and potential speech act verbs. The notion of 'pragmatic weight' is introduced as a label that describes the ways in which subject pronouns are used to signal utterances as 'less abstract' or 'more personally relevant'. Finally, some predictions are made as to what this description of the use of subject pronouns may imply for other, structurally similar pro-drop languages. 
97-270 Egbert, Maria M. (U. of Texas). Context-sensitivity in conversation: eye gaze and the German repair initiator bitte?. Language in Society (Cambridge), 25, 4 (1996), $587-612$.

This article is concerned with the interactive organisation of understanding in conversation, and seeks to demonstrate that, just as turn-taking has been shown to be both context-free and contextsensitive, so too is the organisation of repair. In a comparison of American and German conversation, repair is claimed to be context-free in that, basically, the same mechanism can be found across the two languages. However, repair is also sensitive to the linguistic inventory of a given language; in German, morphological marking, syntactic constraints, and grammatical congruity across turns are used as interactional resources. In addition, repair is sensitive to certain characteristics of social situations. The selection of a particular repair initiator, German bitte?,'pardon?', indicates that there is no mutual gaze between interlocutors, i.e. there is no common course of action. It is claimed that the selection of bitte? not only initiates repair, it also spurs establishment of mutual gaze, and thus displays that there is attention to a common focus.

\section{7-271 Giora, Rachel (Tel Aviv U.) and others. Identification of written discourse topics by structure coherence and analogy strategies: general aspects and individual differences. Journal of Pragmatics (Amsterdam), 26, 4 (1996), 455-74.}

Categorically structured informative texts exhibit their discourse-topic in the beginning. When asked what the text is about, the skilled reader would deeply process the first proposition and skim through the rest for disconfirmation. S/he will, therefore, perform poorly on incoherent texts whose discourse-topic is displaced. Gifted and normal highschool students from a high socioeconomic neighborhood correctly identified more topics in coherent than in incoherent texts (Experiment 1). Low socioeconomic status subjects performed more poorly than the high socioeconomic status subjects on coherent texts, but better on incoherent texts (Experiment 2). Analogy improved performance on coherent texts among low socioeconomic status subjects, who came from academic classes, but did not affect performance on incoherent texts. Experiment 3 studied discourse-topic identification of schematically organised texts by low socioeconomic status subjects, and found that analogies impaired it. The results are discussed in terms of the distinction between general comprehension and text-specific strategies.

\section{7-272 Nelson, Gayle L. (Georgia State U.) and others. Arabic and English compliment responses: potential for pragmatic failure. Applied Linguistics (Oxford), 17, 4 (1996), 411-32.}

This study investigated similarities and differences between Syrian and American compliment responses. Interviews with Americans yielded 87 compliment/compliment response sequences and interviews with Syrians resulted in 52 sequences. Americans were interviewed in English and Syrians in Arabic. Data consisted of demographic information and transcriptions of the sequences. The entire set of data was examined recursively. This examination suggested three broad categories (acceptances, mitigations, and rejections) and subcategories. Two trained raters coded each of the English and Arabic compliment responses as belonging to one of the categories. Intercoder reliability for the American data was 92 per cent and 88 per cent for the Syrian data. Of the American compliment responses, 50 per cent were coded as acceptances, 45 per cent as mitigations, and 3 per cent as rejections. Of the Syrian compliment responses, 67 per cent were coded as acceptances, 33 per cent as mitigations, and 0 per cent as rejections. Results suggest that both Syrians and Americans are more likely to either accept or mitigate the force of the compliment than to reject it. Both groups employed similar response types (e.g. agreeing utterances, compliment returns, and deflecting or qualifying comments); however, they also differed in their responses. US recipients were much more likely than the Syrians to use appreciation tokens, and a preferred Syrian response, acceptance+formula, does not appear in the US data at all. 